\title{
Novel Dressing Method of Fixing Polyurethane Foam on the Soft Tissue Defect of the Finger
}

\author{
Sang Hwan Lee ${ }^{1}$, Seung Hyun Sim${ }^{1}$, Sae Hwi Ki ${ }^{1,2}$, Jong Hwan $\mathrm{Choi}^{1}$, Hyung Mook Kim \\ 'Department of Plastic Surgery, Inha University Hospital, Incheon; '²Department of Plastic Surgery, Inha University School of Medicine,Incheon, Korea
}

\begin{abstract}
Background: In the fingertip injury, there are many reconstruction methods such as skin graft, local flap, regional flap, and free flap. These methods can be performed immediately after injury. But when reconstruction can not be performed immediately, the raw surface of the finger tip is very painful and disgusting. We tried to find new dressing method with good wound care and high patient satisfaction before the surgery.

Methods: From March 2016 to June 2017, 15 patients who acquired a fingertip injury, but could not undergo emergency surgery, were enrolled. A polyurethane foam was fixed to their fingertip damaged area using 6-0 nylon. After one week the fixed foam was removed. From the next day after the foam fixation dressing, subjective pain was checked for one week at two-day intervals and recorded on a visual analog scale (VAS). The formation of granulation tissue and occurrence of infection were checked.

Results: Patients' pain gradually decreased over time. Patients' VAS score was the largest at 7.13 on the next day after the foam fixation dressing, and decreased to 2.72 at one week when the foam was removed. All patients had granulomatous tissue and no infection. Patients were able to successfully perform the fingertip reconstruction with a planned method.

Conclusion: When the patient with fingertip injury cannot be operated immediately, foam fixation dressing can be used before the planned elective surgery. This may help to reduce patient's pain and to obtain good surgical results.
\end{abstract}

Keywords: Finger injury, Polyurethane foam, Wound and injuries

\section{Introduction}

Fingertips are most frequently used in daily life, and more injured site than other parts of the body [1]. In the fingertip amputation, replantation surgery can be performed successfully due to the development of microsurgery [2]. Most of the patients with fingertips injuries come to the emergency room and receive emergency surgery, but some patients cannot be cared for immediately for diverse reasons. Patients who do not bring the amputated part or who are not candidates for immediate reconstruction because of severe injury or contamination, we can use the many methods including stump revision, skin grafting, local flaps, free flaps for fingertip reconstruction [3]. In some cases, reconstruction operation cannot be performed immediately, and meet to be scheduled for elective surgery.

Recently, negative pressure wound therapy (NPWT) has been used to maintain proper moisture and help wound management before elective surgery [4]. However, the fingertip area is not easy to apply because it is difficult to fix it on fingertip wound. Also, there is a disadvantage that patients feel uncomfortable because they have to carry a machine at all times. Furthermore changes of the foam dressing can be painful.

Therefore, we applied a new method with fixing a polyurethane foam on fingertip

\section{Original Article}

Received: September 20, 2017

Revised: September 252017

Accepted: September 272017

\section{Corresponding author:}

Sae Hwi Ki, M.D., Ph.D.

Department of Plastic Surgery, Inha University School of Medicine, 27 Inhang-ro, Jung-gu,

Incheon 22332, Korea

Tel: +82-32-890-3870-3

Fax: +82-32-890-2918

E-mail: mdki1967@gmail.com

No potential conflict of interest relevant to this article was reported.

This is an Open Access article distributed under the terms of the Creative Commons Attribution Non-Commercial License (http://creativecommons.org/licenses/by-nc/4.0/) which permits unrestricted non-commercial use, distribution, and reproduction in any medium, provided the original work is properly cited.

(c) 2017 Korean Wound Management Society 
wound for a week before the elective surgery. The purpose of this study was to investigate the effects of preventing dehydration, the degree of pain reduction, and the presence of infection in patients who applied the foam fixation dressing.

\section{Methods}

From March 2016 to June 2017, 15 patients who received a fingertip site who could not undergo emergency surgery were enrolled. Patients with severe disease, contaminant wound, diabetes, dialysis, and autoimmune diseases were excluded. Imaging studies were performed to confirm that there was no foreign material. After that, digital anesthesia was performed. A finger tourniquet was applied and the wound was cleaned with normal saline. The size of the fingertip injury area was measured and the polyurethane foam was cut according to the measured fingertip wound size. The entire defective area was covered by polyurethane foam and simple interrupted suture was performed using nylon 6-0 for fixing it (Fig. 1A). After applying a finger splint to the damaged finger, the patient was followed up at the outpatient clinic.

During the outpatient follow-up, daily dressing was per- formed around the fixed polyurethane form, and the fixed foam was kept for one week. Patients received oral antibiotics of the 1st generation cephalosporins two times a day during the period. It was planned to remove the fixed foam immediately if any inflammation occurred. On the next day after the foam fixation dressing treatment, the 3rd and 5th day after the treatment, and the 7th day of removing the fixed foam, the degree of pain felt by the patients was measured using a visual analog scale (VAS) score.

The age, sex, fingertip bone exposures, and size of defects were investigated in fingertip injury patients used foam fixation dressing. It was confirmed whether there was any inflammation or infection. The degree of pain felt by patients before the planned surgery was analyzed. When the fixed foam was removed, the formation of granulation tissue was visually evaluated (Fig. 1B).

\section{Results}

A total of 15 fingers were examined. The patient's age ranged from 19 to 76 (mean 46.5 years) and there were 9 men and 6 women. Patients injured their fingertips by a knife or glass.
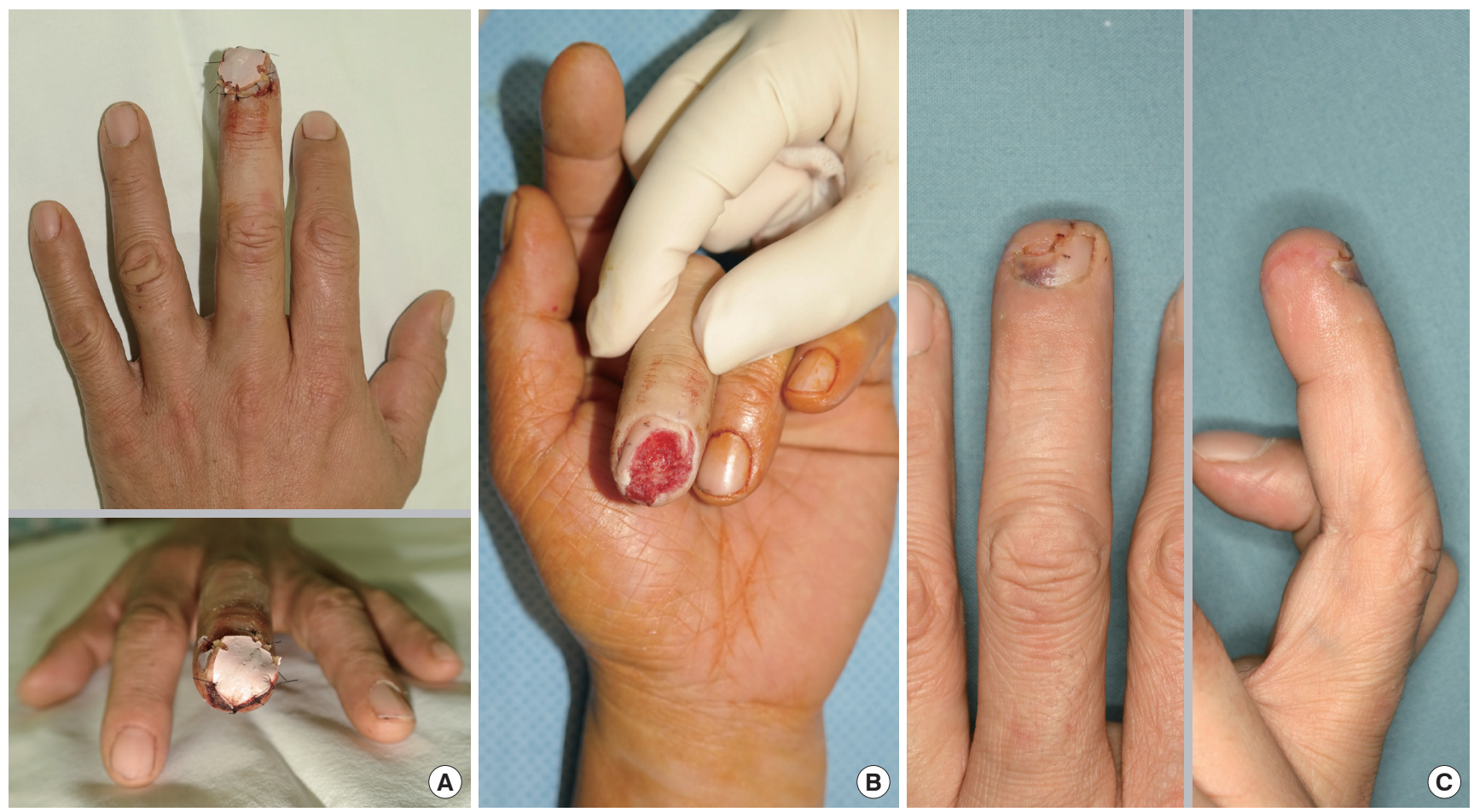

Fig. 1. (A) Polyurethane foam was fixed to the damaged fingertip area using 6-0 nylon at patients who could not undergo emergency surgery. (B) After one week the fixed foam was removed. There was no infection and the healthy granulation tissue formation was seen. (C) Postoperative 1 month photography. The fingertip reconstruction using the $\mathrm{V}-\mathrm{Y}$ advancement local flap was successfully performed. 
The full thickness skin of the damaged fingertip was deficient. The fingertip damage size ranged from $0.5 \times 1.0 \mathrm{~cm}^{2}$ to $2.0 \times 1.5 \mathrm{~cm}^{2}$ and the average size was $1.3 \times 0.8 \mathrm{~cm}^{2}$ (Table 1). There were no cases of inflammation or infection in all patients used foam fixation dressing.

The VAS score, which indicates the degree of pain felt by the patients was average 7.13 on the next day after application of the foam fixation dressing, and decreased to average 5.5 on the third day of the application and average 2.73 on the 7 th day of the application. It tended to decrease over time. The patients with the largest defect of $2.0 \times 1.5 \mathrm{~cm}^{2}$ showed 7 on the next day after the foam fixation dressing and 6 on the third day and 4 on the seventh day. There were two patients with the smallest defect size of $1.0 \times 0.5 \mathrm{~cm}^{2}$. One patient had 7 on the next day, 4 on the third day, 2 on the third day, and 2 on the 7th day. The other patient had 5 on the next day, 2 on the third day, and 2 on the 7th day (Table 2).

When the foam was removed on the 7th day after the foam

Table 1. Patient demographics

\begin{tabular}{rcccccl}
\hline No. & Sex & Age & Injury site & Bone exposure & Defect size $\left(\mathrm{cm}^{2}\right)$ & \multicolumn{1}{c}{ Reconstruction method } \\
\hline 1 & M & 76 & 2nd finger, Rt. & 0 & $1.5 \times 0.5$ & V-Y advancement flap \\
2 & M & 74 & 3rd finger, Lt. & X & $1.0 \times 0.5$ & Split thickness skin graft \\
3 & M & 33 & 3rd finger, Rt. & X & $1.3 \times 0.5$ & Split thickness skin graft \\
4 & M & 63 & 1st finger, Rt. & X & $1.2 \times 0.5$ & Split thickness skin graft \\
5 & M & 38 & 4th finger, Lt. & 0 & $1.5 \times 1.0$ & V-Y advancement flap \\
6 & M & 19 & 1st finger, Rt & X & $1.0 \times 1.0$ & Split thickness skin graft \\
7 & M & 55 & 5th finger, Lt. & X & $1.0 \times 0.8$ & Split thickness skin graft \\
8 & M & 44 & 2nd finger, Rt. & 0 & $2.0 \times 1.5$ & Radial artery superficial palmar branch free flap \\
9 & M & 27 & 1st finger, Rt. & X & $1.4 \times 1.0$ & Split thickness skin graft \\
10 & F & 38 & 4th finger, Lt. & X & $1.2 \times 0.5$ & Split thickness skin graft \\
11 & F & 48 & 4th finger, Lt. & 0 & $1.6 \times 1.5$ & V-Y advancement flap \\
12 & F & 59 & 2nd finger, Lt. & X & $1.0 \times 0.5$ & Split thickness skin graft \\
13 & F & 55 & 3rd finger, Rt. & 0 & $1.5 \times 1.0$ & V-Y advancement flap \\
14 & F & 44 & 3rd finger, Rt. & X & $1.5 \times 0.5$ & Split thickness skin graft \\
15 & F & 25 & 4th finger, Lt. & 0 & $1.2 \times 0.5$ & V-Y advancement flap \\
\hline
\end{tabular}

Table 2. Pain changes in patients with foam fixation dressing according to VAS score

\begin{tabular}{lccc}
\hline \multirow{2}{*}{ Patient No. } & \multicolumn{3}{c}{ Post foam fixation dressing day } \\
\cline { 2 - 4 } & 1 day & 3 day & 7 day \\
\hline 1 & 8 & 8 & 2 \\
2 & 7 & 4 & 2 \\
3 & 8 & 7 & 3 \\
4 & 6 & 3 & 2 \\
5 & 5 & 2 & 1 \\
6 & 6 & 4 & 2 \\
7 & 7 & 7 & 3 \\
8 & 7 & 6 & 4 \\
9 & 8 & 7 & 5 \\
10 & 7 & 5 & 2 \\
11 & 8 & 7 & 3 \\
12 & 5 & 2 & 2 \\
13 & 8 & 7 & 5 \\
14 & 9 & 7 & 2 \\
15 & 8 & 7 & 3 \\
\hline
\end{tabular}

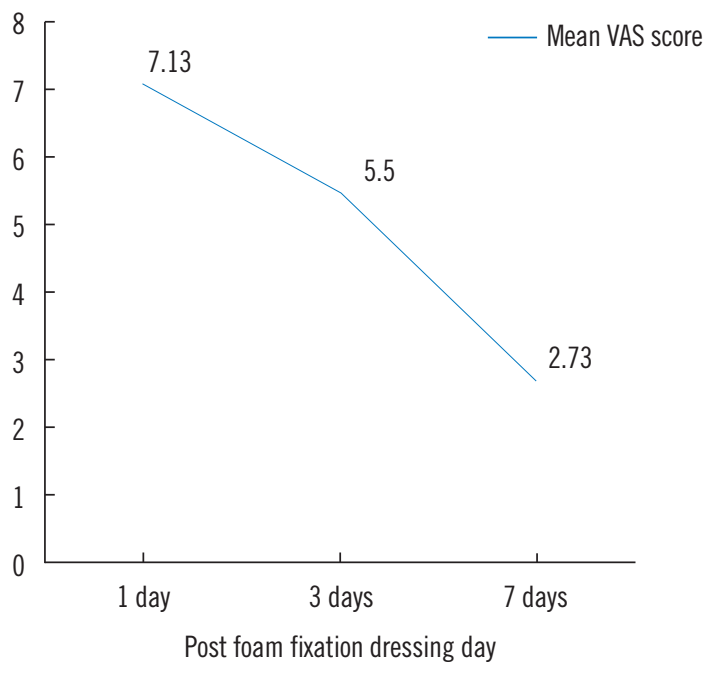


fixation dressing, the formation of healthy granulation tissues was grossly observed in all patients. Patients with partially exposed distal phalangeal bones were also found to be covered with healthy granulation tissues. Nine patients who had no bone exposures on fingertip was undergone the split thickness skin graft after the removal of the fixed foam. Five of six patients who had bone exposures on fingertip was undergone V-Y local advancement flap after the removal of the fixed foam. One patient with the greatest defect of $2.0 \times 1.5 \mathrm{~cm}^{2}$ was undergone a radial artery superficial palmar branch free flap (Table 1). There were no complications in our patients after the fingertip reconstruction surgery (Fig. 1C).

\section{Discussion}

The fingertip is very important area for the pinch and grasp, and, we always use the fingertips in our daily life, such as picking up objects [5]. Inappropriate treatment with fingertips may cause many inconveniences to us. Because fingertips are damaged commonly among the hand injuries, various reconstruction methods have been introduced. Beasley et al. [6] reported that skin grafts can be applied to fingertip injury where distal phalangeal bones are not exposed. If the bone in fingertip is exposed, it can be treated with various local flaps, such as a volar or lateral V-Y advancement flap, cross finger flap [7]. If the fingertips defect is too large to use the previous methods, a island flap could be used [7,8]. Recently, microscopic techniques have been developed and more difficult flaps such as toe-pulp free flap could be performed [9].

Most of the patients with fingertips injuries come to the emergency room and receive emergency surgery. In the case of finger amputation, if the replantation surgery is performed immediately, the best results may be obtained. However, if there is no amputee site, or if it is impossible to do reconstruction surgery immediately, it is important to find out the appropriate treatment method according to the conditions of the patients' injury site. It can be more helpful to preserve the patient's ability to finger function. Several reconstruction methods of the fingertips should be determined by comparing pros and cons. Age, major hand, severity of injury, location of soft tissue defect, presence of bone exposure, willingness and rehabilitation for patient's treatment, and surgical skill of medical staff should be considered.

Patients who cannot undergo emergency surgery will be scheduled elective surgery after outpatient follow up. In this case, there is difficulty in managing the fingertip wound until the elective surgery. Frequently encountered problem is that the patient feels severe pain and fear of bleeding and exposed wounds whenever changing the dressings. The Exposured wound also increases the risk of infection. The authors thought that fixing the polyurethane foam on exposed wound keeps the wet environment and reduces the patients' pain feeling whenever the dressing foam was changed. However, since it was doubtful that the risk of infection would increase, so we have plan to remove the fixed foam immediately whenever any inflammation occurred. The patient VAS score was 7.13 after applying the foam fixation dressing in injury day, but began to decrease rapidly from the next day. When removing the fixed foam at one week, their VAS score decreased to 2.72. There was no significant difference in the pattern of pain when comparing patients with large defects and those with small defects. The pain was subjective and the personal tendency to pain rather than defect size was thought to have had a greater impact.

Generally, when the fingertip area is injured, a foam dressing or wet dressing with gauze for the soft tissue defect is selected to maintain a wet environment, and a secondary dressing is usually demanded for attachment of it. However, some cases showed that the foam was peeled off the wound, and the wound area became dry. The wound without skin barrier is rapidly dehydrated when exposed to air for 2-3 hours, which is known to inhibit proliferation, migration and differentiation of epithelial cells and induce necrosis [10]. It has been also known that preventing dehydration of the wound and maintaining a moist environment reduces cell deformation and allows microvessels to open without shrinking and re-epithelization proceeds rapidly $[11,12]$. By completely fixing the foam on the exposed wound, it was possible to prevent the wound area from being dried and to keep the wet environment properly. When the foam was kept for one week and then removed, healthy granulation tissue formation was observed in all our patients.

Dressing foam manuals are advised to change it in accordance with the amount of exudate and the degree of dirt on the wound [13]. Therefore, after fixing the foam, the patient was carefully examined during the dressing of the peripheral area of foam fixation dressing to see whether inflammation or infection occurred. However, no inflammation or infection occurred in our patients. Foam fixation dressings used by the authors reduce the patient's pain and reduce the fear of dressing change for the patient. Adequate surgical plans could also be established through enough dialogue and consultation 
with the patients. Therefore, appropriate surgical methods could be performed on patients and no postoperative complications were observed.

The limitations of this study are that it is difficult to compare and evaluate the effects of the foam fixation dressing and different various dressing methods on fingertip injury because we only used foam fixation dressing. Also, we used it only to the patients who had no specific disease such as diabetes, connective tissue disease, genetic disease associated with wound healing and relatively clean wound. During the week of fixing the foam, there was no inflammation in all our patients, but it is difficult to provide proper foam fixation period in patients with various other factors that might affect wound infection. Further studies on the comparison of the dressing with other dressing and the foam fixation period will be necessary.

\section{Conclusion}

Most of the patients with fingertips injuries come to the emergency room and receive emergency surgery. However, if it is impossible to do reconstruction surgery right now, it is important to find out the appropriate treatment method according to the conditions of the patients' injury site. Before the elective surgery, using foam fixation dressing can reduce the patient's pain and help the patient to choose the most appropriate surgical method. It can be more helpful to preserve the patient's ability to finger function.

\section{References}

1. Chen CT, Wei FC, Chen HC, et al. Distal phalanx replantation. Microsurgery 1994;15:77-82.

2. Chen CT, Wei FC, Chen HC, Chuang CC, Chen HT, Hsu WM. Distal phalanx replantation. Microsurgery 1994;15:77-82.

3. Russel RC. Finger tip injuries. In: McCarthy JG, editor. Plastic surgery. 3rd ed. New York: WB Saunders; 1990. p4485-7.

4. Vogt PM, Andree C, Breuing K, et al. Dry, moist, and wet skin wound repair. Ann Plast Surg 1995;34:493-9.

5. Ganchi PA, Lee WP. Fingertip reconstruction. In: Mathes SJ, Hentz VR, editors. Plastic surgery. Philadelphia: Saunders; 2006. p.153-71.

6. Beasley RW, Principles and techniques of resurfacing operations for hand surgery. Surg Clin North Am 1967;47:389.

7. Venkataswami R, Subramanian N. Amputation of the fingertip and thumb. Plast Reconstr Surg 1980;66:296-300.

8. Evans DM, Martin DL. Step-advancement island flap for fingertip reconstruction. Br J Plast Surg 1988;41:105-11.

9. Morrison WA. Thumb and finger tip reconstruction by composite microvascular tissue from the toes. Hand Clin 1992;8: 537-50.

10. Hartmann M, Jonsson $\mathrm{K}$, Zederfeldt $\mathrm{B}$. Effect of tissue perfusion and oxygenation on accumulation of collagen in healing wounds. Randomized study in patients after major abdominal operations. Eur J Surg 1992;158:521-6.

11. Vogt PM, Andree C, Breuing K, et al. Dry, moist, and wet skin wound repair. Ann Plast Surg 1995;34:493-9.

12. Dyson M, Young SR, Hart J, et al. Comparison of the effects of moist and dry conditions on the process of angiogenesis during dermal repair. J Invest Dermatol 1992;99:729-33.

13. Thomas S. Foam dressing. J Wound Care 1993;2:153-6. 